\title{
State of the art: micro-nanorobotic manipulation in single cell analysis
}

\author{
Yajing Shen ${ }^{1 *}$ and Toshio Fukuda ${ }^{2}$
}

\begin{abstract}
Single cell analysis is an essential research approach to reveal the secret of life. At such a small scale, it puts forward higher demand on the accuracy of the cell manipulation system. This paper reviews the state of the art of micro-nanorobotic manipulation in single cell analysis. First, the key applications of the micro-nanorobotic manipulation system in single cell analysis are introduced, including the single cell injection, positioning, characterization, assembly, and the development of biomedical device. Then, the current key techniques, challenges, and future trends in micro-nanorobot are discussed from the aspects of actuating, sensing, controlling, system integration, and commercialization. To meet the requirement of cell biology, the next generation micro-nanorobot should have small, automatic, high integration and applicable features in multiscale.
\end{abstract}

Keywords: Single cell analysis; Micro-nanorobot; Micro-nanomanipulation; Biomedical

\section{Review}

\section{Introduction}

The single cell is the smallest unit of living things and is often called the building block of life [1]. This small organizational system maintains a highly complex and hierarchical architecture of interconnected molecular networks and represents life and its related consciousness [2]. The understanding of such a complex system will help us to elucidate the functioning of complete organs and will pave new ways to cure diseases or to develop personalized medicine.

One contribution of single cell analysis relies on the existence of cellular heterogeneity within the same population of cells. Bulk-scale measurements, which rely on data averaged across thousands or millions of cells, report only average values for the population. Thus, the characteristics of a group can obscure the differences between the individuals in it. Because studies made at the single cell level are not subject to the averaging effect characteristic of bulk-phase population-scale methods, they offer a level of discrete observation that is unavailable with traditional biological methods [3]. Secondly, single cell analysis provides an approach to identify the

\footnotetext{
* Correspondence: yajishen@cityu.edu.hk

'Department of Mechanical and Biomedical Engineering, City University of Hong Kong, Tat Chee Avenue, Kowloon, Hong Kong 99907, China Full list of author information is available at the end of the article
}

differences between individual cells. By characterizing the single cells at different conditions, such as health and unhealthy, we can take the single cell as an ideal model to detect disease, test medicines, and develop drugs. Furthermore, analyzing the content within single cells would benefit the deeper understanding of cellular functionalities and behaviors. For example, in the field of immunology, single cell analysis can help us to monitor gene expression in complex tissues, to define different cell subsets, to get insights into both their functional capacity and their specificity, and to track antigenspecific cells [2]. Therefore, nowadays, techniques for single cell analysis have attracted increasing interests in cell biology and the life science.

Single cell analysis is a broad subject that covers many research areas, such as single cell genomics, immunology, diagnostics, and so on. Many review articles have been reported to discuss the problems and trends of single cell research from the biological view [4-8]. This article will not address the above biological problems deeply but will mainly focus on the engineering problems behind those biological issues. To perform single cell analysis, the analysis platform should have both accurate observation and manipulation abilities. For observation, various effective microscopes have been developed, such as optical microscopy (OM), atomic force microscopy (AFM), environmental scanning electron 
microscope (ESEM), scanning ion conductance microscopy (SICM), and so on. Generally, each observation system has its own advantages and issues for the single cell analysis; for instance, OM is easy to use but cannot provide high image resolution, AFM can provide high image resolution but limited to the nonrealtime observation, and ESEM allows real-time high resolution imaging but cannot for in vivo cell research. The review about the observation system for single cell analysis at nanoscale can be found from the article report by Zheng [9]. These different types of observation systems make high demand on the manipulation system correspondingly. Here, micro-nanorobot technique is widely employed owing to its accuracy, controllability, flexibility, and other unique features. Many articles have been reported to discuss the application of the micro-nanorobot in the biomedical field. For example, Dario reviews robotics and microsystems in biomedical applications $[10,11]$, Park reports on the biomedical robot for intravascular therapy [12], Nelson reviews microrobotics for minimally invasive medicine [13], Lenaghan reviews the art and challenge of nanorobot for cancer therapy [14], and so on. For the specific topic of single cell analysis, the micro-nanorobot plays more and more important roles in recent years but few review papers have been reported.

Micro-nanorobotic technique allows the precise manipulation at a small scale. It provides essential engineering support for the biological research at the single cell level, including the cell injection, positioning, characterization, surgery, and so on, which gives us a novel approach to elucidate the fundamental and practical principles within the cell. In this article, we will review the state of the art of micro-nanorobotic manipulation for single cell analysis, including its key applications, main techniques, the current challenges, and the trends in the future.

\section{Key applications}

With the rapid growth of nanotechnology in the last few decades, the techniques for precise positioning and manipulation also have a fast development. Micro-nanorobotic manipulation is an advance method by integrating the traditional robotic technique and micro-nanotechnology together. Generally, the micro-nanorobotic manipulation system allows precise positioning ranged from several nanometers to micrometers and provides flexible movement ability with multiple degrees of freedom (DOFs) in three-dimensional space. In addition, it has high controllability that can automatically perform some tasks based on computer programming. Moreover, the micro-nanorobotic manipulation can integrate with various commercial microscopes, such as OM, SEM, and AFM, to solve different biological problems effectively. Owing to these advantages, micro-nanorobotic manipulation has been widely used in the biological fields. In this section, we will focus on some key applications of the micro-nanorobotic manipulation system in single cell analysis.

\section{Cell injection}

Technologies of delivering exogenous materials into cells play a central role in experimental cell biology, such as cell cloning, DNA therapy, and so on. As one type of nonviral vector-mediated method, micropipette injection has been widely accepted owing to its low toxicity and high delivery efficiency. Microinjection is normally performed with a micromanipulator, which refers to the process of using a glass micropipette to insert into or suck out substances from a single living cell [15-17]. In this process, two key procedures are how to move the pipette to the cell precisely and how to penetrate the cell membrane safely, which directly determine the injection success and efficiency. At such a small scale, it becomes very inefficient and ineffective to perform the injection task by hand directly. Therefore, precise methods based on the micro-nanorobotic manipulation system are widely used. Two main types of injection systems are illustrated in Figure 1.

The glass pipette has been used for drug delivery since several decades ago. Normally, the single cell is handled by a pipette straw and the microinjector is assembled on the micromanipulator. Thus, the position of the micropipette can be controlled via the manipulator precisely, which usually has a positioning resolution higher than $2 \mu \mathrm{m}$ and more than three DOFs [20]. In the traditional methods, the micromanipulator is only taken as a precision tool, which lacks intelligence. To improve the accuracy and to reduce the time cost in the injection process, the automatic injection system is developed based on robotic control technique in recent years. For example, Sun takes the images of the objects, including the cell and glass pipette, as the feedback signals to monitor the injection process dynamically. As a result, an automatic injection process can be realized, which greatly improves the injection success rate and reduces the time cost [18]. In addition, a force sensor is used to measure the penetration force, which is taken as the feedback to ensure the injection safety and improve the injection efficiency. For instance, Xie proposes a twoloop force control framework to regulate the penetration force in the fish embryos injection [21]. Yanagida proposes a piezo-micromanipulator to prevent the large deformation of the cell during the injection [22]. It utilizes the rapid deformation of a piezoelectric element to realize smooth insertion in intracytoplasmic sperm injection, which reduces the penetration force and improves the survival rate. Tan reports a mechanical model to elucidate the cell deformation in the penetration process, 


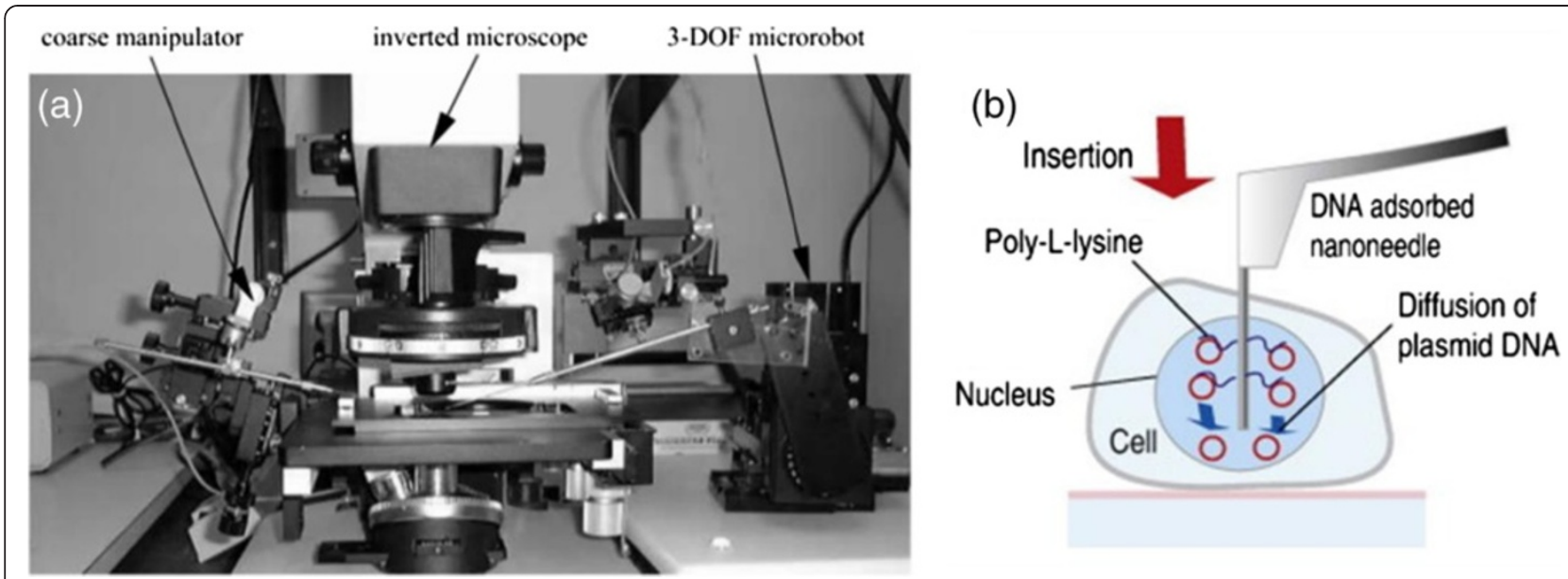

Figure 1 Micro-nanorobotic manipulation system for single cell injection. (a) Autonomous embryo injection system by glass micropipette [18]. (b) Schematics of an AFM cantilever-based nanoinjection [19].

which paves a way to optimize the injection process theoretically [23]. The usage of the robotic technique, including image processing and force feedback, could improve the traditional injection system greatly. However, there are still some intrinsic problems. One serious problem is that the injection is performed under OM, which has an imaging resolution limitation (approximately $200 \mathrm{~nm}$ ); therefore, it makes challenges to the image processing. The limited visual range of the microscopy is another challenge to the micro-nanorobotic system. It is clear that a wider visual range will help us to find the object easier, but the low magnification image will result in the loss of image details. Even though the automatic single cell injection has been realized, this technique is usually performed under an invariable magnification. Integrating the automagnification technique with the micro-nanorobotic manipulation technique will improve the productivity, which should be considered in the future.

In addition to the glass pipette, nanoinjection methods based on an AFM cantilever nanoprobe are also proposed recently $[19,24]$. In these techniques, a smaller injector with nanometer size is used to penetrate the cell and deliver the materials into the cell. The manipulation strategy of the nanoneedle is similar to that of the glass pipette for single cell injection. However, there are few automation techniques reported for the nanoinjection process. We think one main reason is that the nanoinjection technique is still at the stage of laboratory research; therefore, it does not have an urgent demand on the automatic system. However, in the long term, the improvement of the nanoinjection system is important for practical applications.

Consequently, micro-nanoinjection is a simple process in which a needle penetrates the cell membrane and/or the nuclear envelope. The micro-nanorobotic manipulation system provides an effective platform for the injection process. Comparing with manual injection, a robotic manipulation system has the advantages of high quality, productivity, and repeatability. In the future, a commercialized automatic-injection equipment that allows a user to operate it easily without specialized training is expected to be developed.

\section{Cell positioning}

Cell positioning is an essentially important technique, since it is the basis of cell array and single cell analysis in biomedical research. One of the main challenges for cell positioning is how to transfer a single cell to a certain place precisely and effectively. Nowadays, to address the above issues in cell positioning, several micronanorobotic manipulation systems have been proposed. Figure 2 illustrates some micro-nanorobotic manipulation systems for single cell positioning.

One direct method for cell positioning is based on microrobot and microtools. In this design, the microtools, such as the micropipette and microgripper, are assembled on the microrobotic manipulation system. The microtool has the capability to handle and release the cell and the microrobot can control the position of the microtool precisely. Therefore, this integrated system can pick up the single cell and place it on the desired position $[25,30,31]$. To improve the accuracy and reproducibility, force feedback and version feedback are also employed in the cell positioning process. For example, Kim reports a nano-Newton-force-controlled micromanipulator for the soft object manipulation, which can detect the contact force fast and handle the object more safely [32]. Fatikow proposes an automatic control frame for the micro-object manipulation and positioning based on vision feedback, which greatly improves the working efficiency [33]. The microtool manipulation approach 

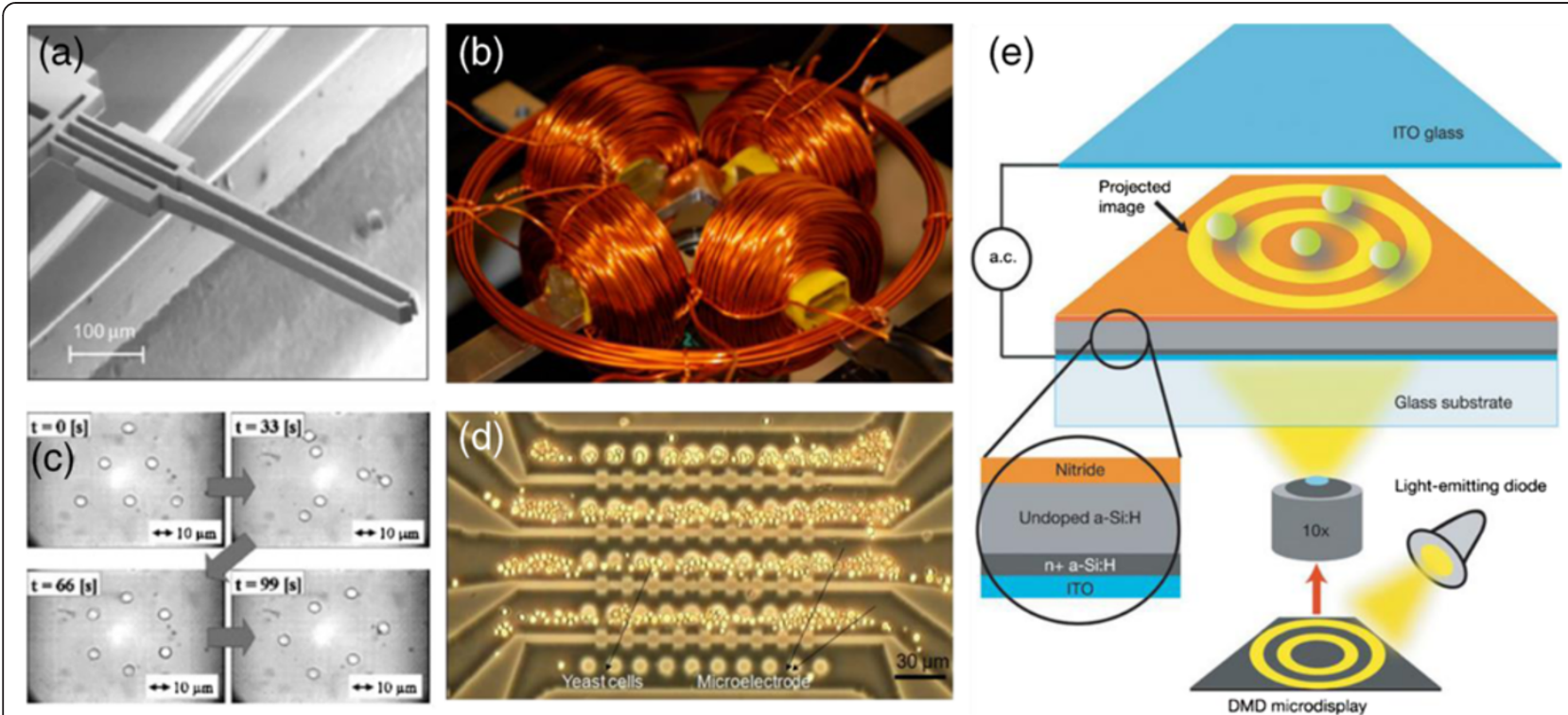

Figure 2 Micro-nanorobotic manipulation system for single cell positioning. (a) Scanning electron micrographs of a microgripper [25]. (b) Five electromagnetic coils are mounted on an optical microscope to actuate the magnetic robot in the center [26]. (c) Microbeads dancing controlled by the optical tweezers [27]. (d) Cell positioning by dielectrophoresis (DEP) chip [28]. (e) Schematic drawing of the optoelectronic tweezers (OETs) [29].

enables the pick-transport-place of a specific single cell precisely and effectively. However, the issue of this method lies in that the microtool has to contact with the cell during the manipulation process. Therefore, it is difficult, if not impossible, to implement this platform in a closed environment, as in the microfluidic channel.

A magnetic robot is actuated by the magnetic field remotely, by which it avoids the usages of electric wires [26]. Therefore, it is able to manipulate and position cells in both open and closed environment. In this kind of manipulation system, the cell movement is usually achieved by the pushing action of the robot. Therefore, this method has a higher requirement on the control system. We have to control the location of the robot and the pushing direction dynamically, which is not convenient for flexible manipulation, especially for the mass cell manipulation.

Optical tweezers are instruments that use a highly focused laser beam to hold and manipulate a small object. In the late 1980s, Arthur and Joseph demonstrated the first application of optical tweezers to the biological sciences, using it to trap an individual tobacco mosaic virus and Escherichia coli bacterium [34]. Today, optical tweezers have been a comment scientific instrument in trapping cells to study a variety of biological problems [35-37]. Fukuda develops a microrobotic manipulation system based on the controlling of the light path of the optical tweezers. It is able to manipulate small objects in 3D space flexibility, such as microbead and single cell [27]. Sun studies the dynamics behavior of the cell in the optical tweezers manipulation and then designs a closedloop control algorithm to transport a single cell or multicells to a certain place [38]. Because optical tweezers can manipulate the object precisely without physical contact, it is very powerful for the cell positioning inside the microchip. However, the main drawback of the optical tweezers is that the generated force by laser is usually limited to nano-Newton, which is not large enough to drive the nonsuspending cells sometimes.

Dielectrophoresis (DEP) is the phenomenon in which a force is exerted on a dielectric particle when it is subjected to a nonuniform electric field [39-41]. DEP has the advantages of noncontact manipulation, good controllability, and high-integration ability within the microchip. Therefore, it has been widely used for cell trapping and sorting, especially for the cell analysis in the microfluidic chip $[28,42]$. One problem of DEP lies in that the strength of the force strongly depends on the medium and particles' electrical properties, on the particles' shape and size, as well as on the frequency of the electric field. Another problem is that it is difficult to make the size of the electrode to nanoscale, which affects the manipulation accuracy.

To overcome the drawback of DEP, especially to avoid the electrode fabrication, a new concept called optoelectronic tweezers (OETs) is proposed [29]. OETs use projected optical images to grab and corral tiny particles with sizes ranging from hundreds of micrometers to tens of nanometers. In this method, light first creates some 'virtual electrodes' on the substrate. Then, the image in 
conjunction with an externally applied electrical bias creates the localized DEP traps in the illuminated areas. It combines the advantages of optical tweezers and electrode-based DEP, which is expected to have a bright future.

As a summary, cell positioning is a very important process in biomedical research. Nowadays, many different types of manipulation strategies have been proposed to deal with this problem. Each method has its own features that can be applied for some certain cases efficiently. Understanding their advantages and drawbacks is very helpful for us to choose the proper approach and develop new methods for the single cell analysis. Considering that the on-chip analysis is more and more important nowadays, a micro-nanorobotic manipulation system that allows cell positioning in a closed environment will be a trend in the future.

\section{Cell characterization}

Single cell characterization provides a quantitative method to study the cell's behavior and to elucidate the cell's activate. For example, the mechanical properties of the single cell can reflect the cell's healthy condition, which benefits disease diagnosis and drug testing [43]. The electrical property of the single cell can reflect the open or close status of the ion channels of the cell membrane, which benefits the understanding of cell transportation [44]. In single cell characterization, micronanorobotic manipulation plays an important role owing to its flexible manipulation capability. Many types of micro-nanorobots and micro-nanotools are used to handle, manipulate, and characterize the single cell to obtain various types of properties of the single cell, including strength, stiffness, resistance, and so on, as illustrated in Figure 3.

The cell's activities, such as mitosis, morphogenesis, cell orientation, cell motility, and survival, depend on the attachment to the extracellular matrix or neighboring cells. Characterization of adhesion strength at the single cell level provides an effective approach to elucidate these activates. Micropipette aspiration is one traditional technique for cell-cell adhesion study [48]. In this method, two aspiration pipettes are assembled on two micromanipulators. Then, the pipettes are used to handle and separate two single cells driven by the micromanipulator. In this process, the cell-cell adhesion strength can be calculated from the suction pressure applied through the pipette. The AFM system is another powerful system for the single-cell-adhesion characterization, which enables to measure the cell-substrate or the cellcell adhesion force. For example, one common method is to put the cell on the AFM sample stage, which is taken as the micromanipulator $(x-y-z)$. Then, the cell is moved against the AFM cantilever driven by the stage, and the adhesion strength can be measured from the deflection of the AFM cantilever [49]. Puech reports a method to measure the cell-cell adhesion strength [50,51]. Here, one cell is immobilized on a modified AFM cantilever tip by chemical glue. Then, the adhesion force between this cell and the other cell on AFM sample stage is obtained by separating these two cells. The AFM system also allows the measurement of the adhesive molecules on the cell membrane [52,53]. Comparing with the aspiration pipette, the AFM system has the advantages of higher positioning accuracy and higher force resolution. However, one drawback is that the manipulation depends on the movement of the AFM sample stage, which is not as flexible as end-effector manipulators. Another issue is that the AFM system cannot scan the sample to get a high resolution image during the characterization process. Environmental SEM (ESEM) is one special type of SEM that can maintain a wet environment within the sample chamber, which provides a novel platform for single cell analysis. Shen reports a single-cell-adhesion study system based on the nanorobotic manipulation system inside ESEM, which can measure both the cell-cell adhesion and cell-substrate adhesion strength [45,54-56]. This manipulation system
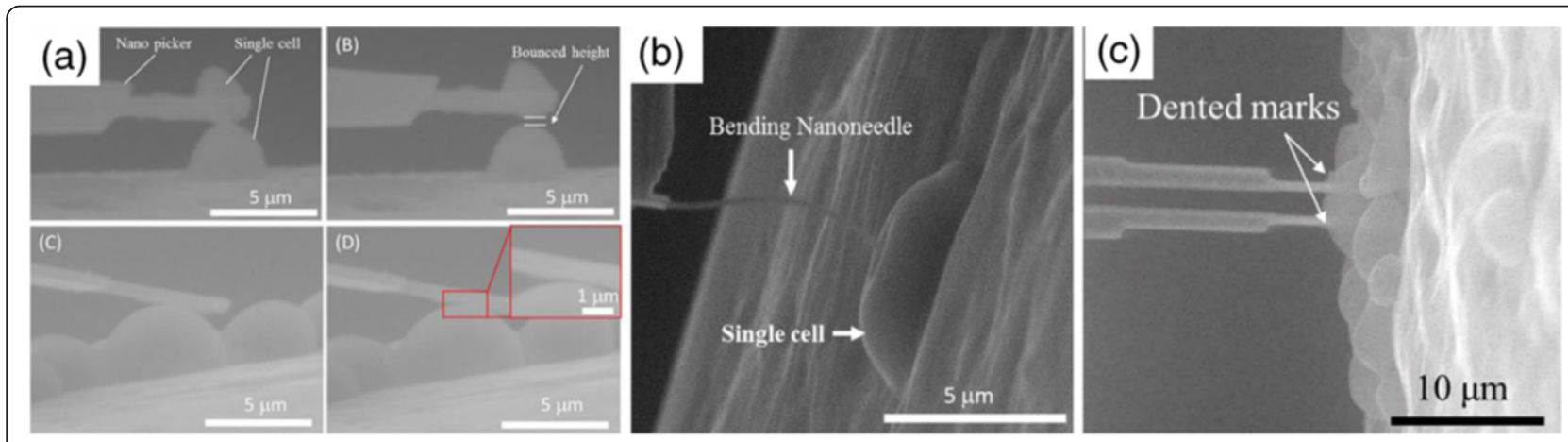

Figure 3 Examples of the single cell characterization by micro-nanorobot. (a) Cell-adhesion-force characterization [45]. (b) Cell mechanical property characterization [46]. (c) Cell electricity characterization [47]. 
has seven DOFs in total and has the positioning resolution approximately $30 \mathrm{~nm}$. More important, this system allows the real-time imaging at nanometer resolution during the manipulation and characterization. These features could provide more information in the characterization process, which could benefit the dynamic single cell study in the future.

Single-cell-mechanical-property characterization, such as stiffness and elasticity, is another example that benefits from micro-nanorobotic manipulation system. Cell stiffness value closely relates to the structure of the cell's membrane and the cytoskeleton within the cell. Therefore, the stiffness of a single cell reflects the cell function in many aspects $[57,58]$. For example, the stiffness of the mammalian cell shows obvious difference after medical treatment or after death, which could benefit disease diagnosis, drug development, and disease therapy [59-61]. Recently, various methods have been reported to characterize the single cell's stiffness based on micro-nanorobotic manipulation technique, including micro-electromechanical systems (MEMS) tool, optical tweezers, magnetic beads [62,63], AFM system, ESEM nanotool, and so on. Nelson assembles a MEMS probe on a microrobotic manipulator to measure the stiffness of the pollen tube, where the MEMS probe is taken as the force sensor and the microrobotic manipulator is used to control the position of the probe [64]. Dao studies the mechanics of the human red blood cell by optical tweezers [65]. Optical tweezers enable high positioning accuracy and high force resolution for the stiffness characterization. However, the relative low force ability (approximately piconewton), the light-induced damage, and the observation accuracy (approximately $200 \mathrm{~nm}$ ) are thought as the challenges for optical tweezers. Magnetic manipulation takes usage of the magnetic field to drive a small bead to deform the cell, which avoids potential lightinduced damage as in laser trapping. Bausch measured the local viscoelasticity of the surface of adhering fibroblasts by controlling the position of the magnetic beads via magnetic fields [63]. The modeling and controlling of the nonhomogeneous magnetic field are considered as the challenge to get accuracy data for the magnetic manipulation method. The high force sensitivity of the AFM system makes it particularly appropriate for studying cell stiffness [66-68]. Here, the AFM cantilever is used as a nanoindentor to deform the cell. As a result, the stiffness can be calculated based on the cell deformation and the applied force $[69,70]$. Despite the AFM technique having been welldeveloped and having shown to be noninvasive to the cell if applied correctly, one existing issue for the AFM cantilever is that the deflection of the cantilever beam may cause slipping on the cell surface during the measurement process. Shen reports a nanoneedle to overcome this drawback [71]. They used the nanorobotic manipulator to control the movement of the nanoneedle with nanoscale resolution inside an ESEM chamber, which allows the characterization of the local region of the cell surface. Consequently, the application of micro-nanorobotic manipulation has enhanced the single cell stiffness characterization greatly. However, the aim of the research is to exactly elucidate how the stiffness value relates to the active cells. From the mechanical information, we expect to know how healthy the cell is rather than if the cell is alive or not. This requirement is bringing new challenges to the current micro-nanorobotic manipulation system, i.e., much faster, more accuracy, and more intelligent.

Biological cells, which consist of a conductive saltwater interior coved by a relatively insulated outer sheath, continuously exchange ions with surroundings. Therefore, the electrical property of a single cell can reflect the physiological state of the cell. Nowadays, the characterization of a cell's electroconductivity has attracted increasing interest in the cell viability identification, ion channel monitoring, drug testing, and other biological fields. Ahmad designs a dual-probe and integrates it with a nanorobotic manipulation system [47]. This system is used to measure the electrical resistance of the single cell, and the results clearly show the variability between living and dead cells. Zhang studies the electrical response of the human embryonic kidney cell corresponding to external mechanic stimulation based on a robot-assisted AFM manipulation system and a patch-clamp system, which can monitor the ion transfer through the ion channels in the cell membrane [72]. This system has been expected to test the effectiveness of drugs in the future. Although micro-nanorobotic manipulation allows the characterization of the single cell electrical conductivity, there are still some challenges. For example, monitor of ion transfer in one single ion channel is still difficult, since it requires higher imaging, positioning, and sensing ability to the robotic manipulation system. Another challenge is the high throughput, i. e., how to find the regions and characterize the electrical property of the single cell faster, which has a higher requirement on the robot intelligence and automatics.

As a summary, micro-nanorobotic manipulation is the essential technique for accuracy positioning and manipulation. It has been widely used in single cell characterization, including the cell's mechanical property, electrical property, its interaction with the environment, and other features. In the future, the micro-nanorobotic manipulation system should be more accurate and intelligent to provide more information for single cell analysis. 


\section{D cell assembly}

As the basic unit of life, the single cell is the elementary block to construct tissues and organs, which show complex structures in three-dimensional space. It is clear that cell culture in $3 \mathrm{D}$ space is much more close to the practical comparing with the traditional 2D plate culture. Many researches have also proved that the cell growth in 3D space has a different behavior with that in the $2 \mathrm{D}$ plate, such as the cancer cell [73]. Moreover, the $3 \mathrm{D}$ cell culture is also an essential technique to develop the artificial organs. For example, an artificial ear has been created successfully by culturing the cells on a 3D scaffold [74]. Therefore, recently, increasing interests have been focused on how to build a 3D structure from a single cell. This research topic, called as ' $3 \mathrm{D}$ cell culture', is quit broad that covers many research areas, including but not limited to cell culture, growth, physiology, and so on (Figure 4a) [75]. In this review, we will not discuss the biological problems but will discuss this topic from the engineering point of view, i.e., '3D cell assembly'. It is subject to '3D cell culture', but specifically focuses on the 3D manipulation and assembly methods, in which micro-nanorobotic manipulation involves deeply.

One widely used method for $3 \mathrm{D}$ cell assembly is to fabricate a biocompatible 3D 'scaffold' to support the growth of the cell [78]. Thus, the key issue becomes how to fabricate the 3D scaffold with an accurate structure. One main scaffold fabrication technique is based on 3D printing. Here, the scaffold material is deposited layer by layer controlled by a flexible manipulation system. For example, Yan manufactures 3D architectures through a controllable manipulation system with three DOFs $(x-y-z)$ and successfully uses it to study the growth of hepatocytes. Klein fabricates one type of two-component polymer scaffolds through laser, directly writing based on nanomanipulation [76]. This scaffold is successfully applied to study the growth of a single fibroblast cell in $3 \mathrm{D}$ space. In addition to $3 \mathrm{D}$ printing, another scaffold fabrication technique is also proposed based on physical or chemical reaction. For example, some materials are sensitive to light; that means the biocompatible liquid will be solidified after exposure to light. Based on this feature, the 3D scaffold can be fabricated by controlling the projection area of the light. For example, Liu reports a method by light-induced gel to generate a 3D scaffold, where the shape and height of the gel can be controlled precisely by controlling the light illumination area and the exposure time [79].

Another assembly method is to build the small blocks with cells into a complex $3 \mathrm{D}$ structure, as building a house from blocks. In this kind of approach, the biological cells are first capsulated in series gel blocks by biochemical process. Then, a microrobot is used to manipulate these blocks to build the $3 \mathrm{D}$ structure. In this method, it is able to change the shape of the structure easily. For example, Tasoglu reports an approach that uses a magnetic crawling microrobot to assemble cellencapsulating gel blocks into a 3D structure, by which various structures can be built from the same series of blocks (Figure 4b) [77].

The $3 \mathrm{D}$ cell assembly is a relative new research area that is rising with the fast growth of the tissue engineering and biomedical engineering research area. Many assembly techniques are benefited from the micronanorobotic manipulation system more or less. However, the final goal of $3 \mathrm{D}$ cell assembly is to construct the functional artificial structures, which are able to replace
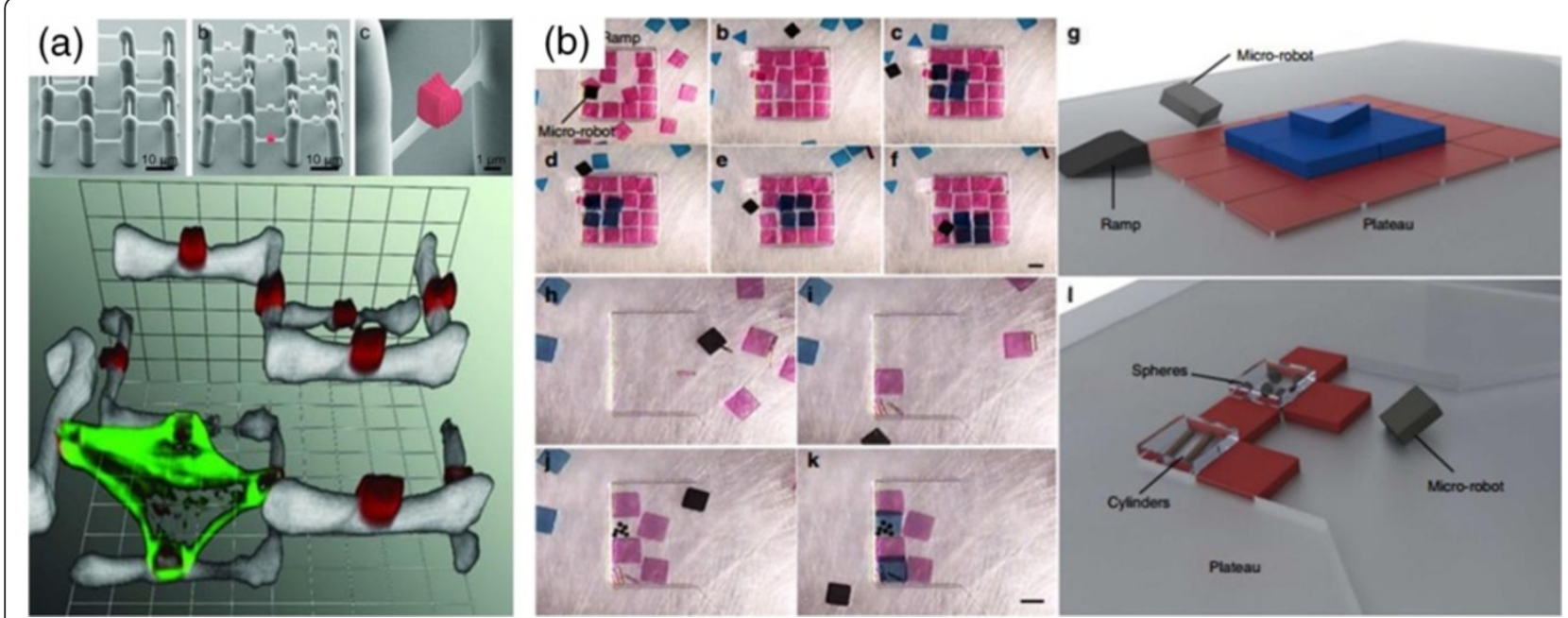

Figure 4 Examples of the 3D cell assembly based on micro-nanorobotic manipulation. (a) Scaffold for single cell 3D growth study by 3D printing [76]. (b) 3D Cell assembly by microrobot [77]. 
the natural tissue and organ. Therefore, it is important to find the way to assemble different types of cells together, which puts forward higher requirement on the intelligence and flexibility of the micro-nanorobot.

\section{Biomedical device}

Micro-nanorobotics is not only an essential supporting technique for single cell research but also could be a miniature device for biomedical application directly. For instance, the endoscopic capsule robot is one of the most popular miniature devices based on robot technique. It integrates the actuating, sensing, and controlling system in one capsule within tens of a millimeter [80]. This kind of robot can not only take images inside our body but can also address the diagnostic or treatment issue automatically. However, these techniques are still difficult to apply at the single cell level owing to the small size of the cell. Zhang reports an artificial bacterial-inspired robot. This robot is fabricated from thin films of semiconductors and its movement is controlled by a magnetic field [81]. The length of this small robot is only several tens of a micrometer, which is within the same scale of a single cell. It has the ability to move outside or inside the human body, which can be used for an invasive medical operation in the future. However, these techniques are not yet mature nowadays, because the robot has to face the actuating and sensing challenges within a small space. The development of small biomedical robot that can work as an independent device will be an essential step for the practical applications for single cell analysis. In the future, we should think of a way to make the robot much smaller, have higher integration, and more functionality.

\section{Current techniques, challenges, and future trends Actuating}

A large type of the micro-nanorobotic actuating depends on the mechatronic principle, such as hydraulic motor, electric motor, electrostatic actuator, piezo actuator, and so on. Hydraulic and electric motors have the ability to generate a large force output and provide long movement distance. However, they cannot provide accurate positioning resolution at nanometer scale. Therefore, they are usually taken as the stage to drive the micronanomanipulator mode coarsely. Electrostatic actuation is one widely used technique in the development of MEMS device, which allows both the precise actuating and sensing. For example, many micro-nanomanipulators, like the microgripper, are designed based on electrostatic mechanism $[82,83]$. However, the short moving distance is one problem for electrostatic actuation, which is usually less than several millimeters. Therefore, these kinds of manipulators are usually taken as the end effector for the micro-nanorobot. Piezo actuation is an effective approach that converts electrical power to mechanical movement (Figure 5). It can provide a large movement distance with several centimeters and a positioning resolution as high as several nanometers. Nowadays, the piezo-based manipulator has been well developed and available in market. The mechatronic-principle-based manipulator has a relatively mature physical model; therefore, it is easy to control and implete. One main issue for this type of actuation is the existence of wires, which make it not applicable for work in a closed environment, such as inside a microchip or inside our body.

Wireless actuation-based manipulation avoids the usage of electrical wire to connect the power supplier. Therefore, it provides the possibility to work in a closed environment. The commonly used wireless actuation techniques include optical tweezers, DEP, magnetic driving, and so on. As discussed in the 'Cell positioning' section, these actuators are driving by physical fields, like light field or electromagnetic field. Thus, they can manipulate the object in a narrow space with high positioning accuracy, which are very important for the in situ analysis in the microchip. For the wireless actuation, the controllability and repeatability are big challenges, since it is difficult to build an exact physical model to control the statues of the small object in the fields. Another issue is that the force generated by these methods is usually smaller than the mechatronic actuation due to the physical principle.

In addition to the above two types of actuating methods, the biochemical actuator also receives more and more attentions nowadays. In these techniques, creatures or part of creatures are taken as the actuator directly, such as sperm, bacterial, flagella, protein, DNA, and so on, which have the moveable ability naturally. If a proper stimulation is given, the movement of these biorobots can be controlled effectively. For example, Nogawa

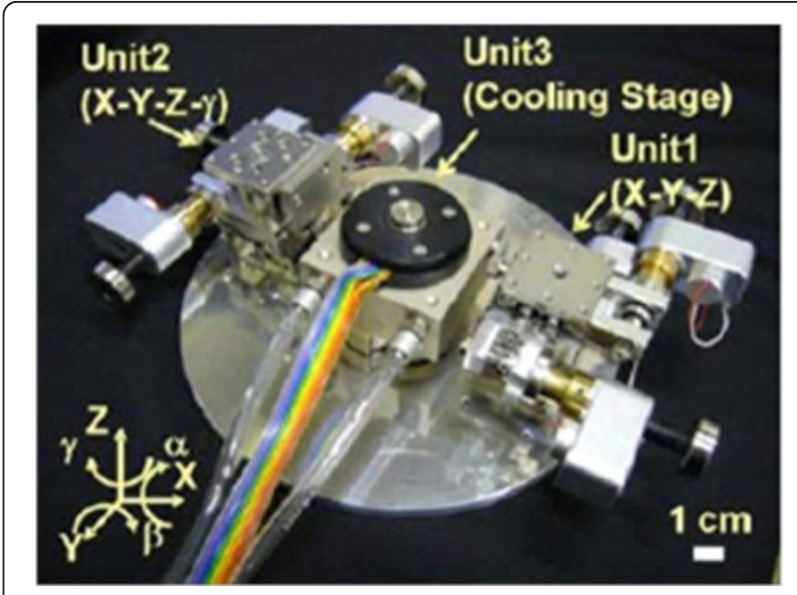

Figure 5 A typical micro-nanorobotic manipulation system based on piezo actuation. 
uses the ion concentration to control the movement direction of bacterial, which can be used as a robot to deliver drugs [84]. Martel uses magnetic field to control the movement of the flagellated magnetotactic bacteria, which can perform the medical operation in the human microvasculature [85]. Scientists also design many prototypes of the nanorobot based on the unique features of protein or DNA, which can work well in a natural biochemical environment and show its significant potential in the drug encapsulation, drug delivery, and disease therapy [86-89]. These biochemical nanorobots have many advantages, such as small size, simple structure, self-assembly, biocompatible, and so on. The problem is that these types of robot are usually sensitive to the environment, such as the temperature, $\mathrm{pH}$ value, chemical solution concentration, and so on. Nevertheless, natural creatures provide us a good example to learn from, which usually have high efficiency and can work better in a biological environment. We believe the biochemical-inspired micronanorobot should be a new trend in the future, especially in the biomedical field.

As a summary, actuation is one of the most important techniques for micro-nanorobotic manipulation, which involves power supply, energy transmission, driven approach, and so on. The current actuating techniques have provided many solutions for the single cell analysis. However, there are still some challenges that need to be addressed, including the optimization of size, working time, output capability, efficiency, and so on. The biological sample is the nanorobot in nature, which has a high working efficiency and is exactly compatible for bioapplication. 'learn from nature' will drive new inspiration in the micro-nanorobot development, which is expected to benefit the biomedical field in the future.

\section{Sensing}

Unlike the traditional robot sensing, only a few sensing technologies can be used at the micro-nanoscale owing to the specific working environment. Because single cell analysis is usually performed under microscopy, the micronanorobotic manipulation has to be workable under microscopy. In this specific case, vision and force are the most effective sensing methods.

In vision sensing, the sample images are captured dynamically from the microscope, firstly. Then, the objects are identified and their positions are calculated based on image processing. After that, the position information is taken as the feedback signal to control the movement of the micro-nanomanipulator. This kind of sensing and control strategy has been successfully used for automatic single cell injection to improve the injection efficiency and reduce the injection time (Figure 6a) [90]. One challenge for the vision feedback lies on the quality of the microscope image. The microscope images usually can only provide a few information about the environment due to the small observation area. Moreover, depth information is hard to obtain from the microscope image directly. Therefore, the object's identification and positioning becomes much more difficult than that at the macroscale.

Force sensing is usually used with vision sensing together to improve the performance of the micronanorobot. For example, in single cell injection, the force feedback has been applied successfully to improve the injection process (Figure $6 b$ ) $[21,91]$. The challenge for force sensing lies on the lack of force/torque information from different directions. Generally, six DOFs, three force and three torque, are required to describe the status of one point clearly. However, it is quite difficult for the force-sensing system at micro-nanoscale, which usually can only provide one to three DOFs.
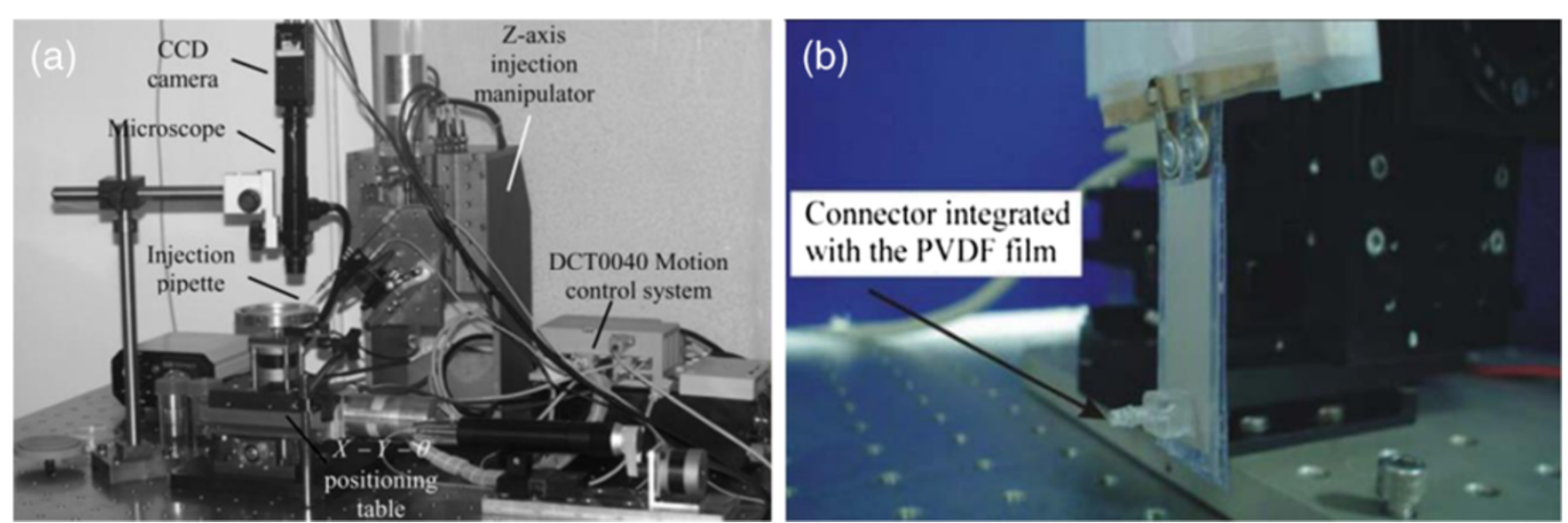

Figure 6 Examples of the sensing system for a micro-nanorobot. (a) A vision feedback system based on the CCD camera for single cell injection [90]. (b) A force feedback system based on a PVDF (Polyvinylidene fluoride) piezoelectric polymer film for single cell injection [21]. 
Therefore, the development of a MEMS force sensor that can work well under microscopy is urgently demanded for the micro-nanorobot sensing.

\section{Control}

At micro-nanoscale, the surface force rather than the inertia force becomes dominate, fluids appear as viscous as molasses, and Brownian motion makes everything incessantly shake [92]. These unique physics make the behavior of the object become sophisticated under manipulation. For example, in the single cell gripping process, the cell may adhere to the gripper surface due to the electrostatic force rather than the clamp force, and therefore, the cell cannot be released after the gripper is opened. Thus, the modeling of the control object becomes a big challenge, which is usually nonlinear, dynamic, randomness, and sometimes even unpredictable. In addition, only a few feedback-sensing techniques, visual and force, can work at the micro-nanoscale. Therefore, controlling is a big challenge for the micronanorobot owing to the modeling and sensing problems.

Nowadays, the micro-nanorobot can perform some simple tasks automatically, for example, the single cell injection. Here, the mechanical behavior of the cell can be modeled, so the traditional visual feedback, force feedback, with PID controller, and impedance control strategy can be used (Figure 7). However, in most of the cases, the single cell analysis requires complex manipulation at a much smaller scale. It is hard to build the physical model to describe the controlled objected exactly. Thus, most of the control process has to be performed based on the master-slave manipulation, in which the decisions are made by a human rather than the robot itself.

Consequently, the main difficulty for the micronanorobotic control is not the control algorithm but lies in the exact modeling of the controlled object. We must consider the unnormal physical phenomena at a small scale. In addition, the lack of powerful feedback signals is also considered as a drawback for the micro- nanorobot system. In future work, molecular dynamics modeling or quantum electrodynamical models should be considered to better describe the property and behavior of the object at small scale. In addition, the small and intelligent sensor for micro-nanorobot should be another essential way to solve current control problem.

\section{System integration}

The micro-nanorobotic manipulator has the ability to move in micro-nanometer scale accurately, but it does not mean the size of the robot system itself is within micro-nanometer size. Actually, to the contrary, a large space is always required to support the robot to work well, since all the driving, sensing, and controlling modules need space to put equipment. However, on the other hand, we expect the robot can be as small as possible to improve its movability, especially its working ability in a closed environment. Therefore, system integration is a big issue in micro-nanorobot design.

Various integration methods have been reported to address different kinds of problems in single cell analysis. For the research in an open environment, such as single cell injection and mechanical characterization, it is easy to find extra space for the auxiliary equipment. The micro-nanomanipulators can be fixed on the microscopy stage and connected with the auxiliary equipment in other places by electrical wires. Contrarily, for the single cell analysis in a closed environment, it is difficult to connect wires directly. In this case, wireless actuation methods are usually used, such as optical tweezers and DEP. However, the wireless connection method brings difficulties to force sensing, because there is no force sensor and the force has to be estimated from the deformation of the object or the applied voltage indirectly. The lack of force information in this integration system increases the control difficulty to the micro-nanorobot. Arai reports a magnetically driven microtool (MMT) chip by introducing the MEMS technique into the robot [94]. They fabricated the magnetic-sensitive microtool based on MEMS technique. Then, they take it as the

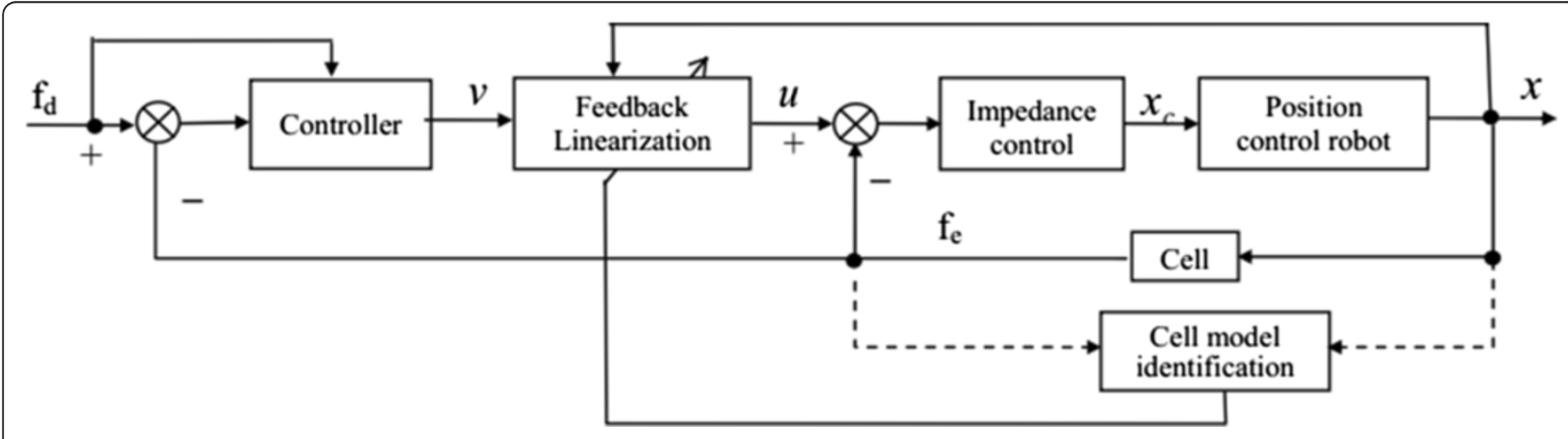

Figure 7 An example of a force feedback control diagram for single cell injection [93]. 
end effector of the robot and assemble it inside the microchip. This kind of robot can work inside the microchip since it can be controlled by the magnetic field remotely. Furthermore, it has the sensing ability owing to its deformable structure. The integration of the MEMS technique and robot technique will promote the micronanorobot research. We think this kind of method will be a trend for the single cell analysis in the future.

The micro-nanosystems mentioned above can be roughly divided to two parts, i.e., the functional end effector and other auxiliary parts. They communicate with each other either by wire or wireless field. How to integrate them together into a whole system is a big challenge. If we take a look at the internal of creatures, we can find many sophisticated robots in nature. Therefore, we think in addition to the physical way, the biological and chemical principles may also be helpful to design a novel micro-nanorobot. For example, the bacteria themselves have the actuating and sensing abilities and even have their own intelligence. Nowadays, artificial bacteria have been designed based on bacteria's actuating mechanism, which can be injected into the body and swim driven by magnetic field. DNA is another excellent robot mode for us. Scientists have developed the walking robot, DNA computer, and DNA cages by programming, which are the first crucial steps towards the small micro-nanorobotic systems.

Consequently, the next generation of the micronanorobot will require on-board sensors, functional tools, computing components, and so on. Combining the robot manipulation technique and MEMS fabrication technique and referring the biological and chemical technique could be the way to create a much smaller and highly integrated robot in the future.

\section{Commercialization}

One significant goal of the micro-nanorobotic research is to solve problems at the single cell scale and finally benefit society. Therefore, how to make the micro-nanorobot system more acceptable should be considered, especially for the acceptance by the biological scientist and the biological company. The automatic single cell injection system is only a handful of successful commercial systems that use micro-nanorobots. However, currently, most of the commercialized micro-nanorobots are only at the stage of precision manipulators. They are able provide accurate positioning capabilities but cannot solve a specific biological problem directly, which are still far from commercialization. Nowadays, most of the application research are still at the laboratory stage, which mainly focuses on the reliability but without considering the cost and productivity. From the commercial point of view, the system should be reliable, low cost, and high production.
Thus, in the future research, these aspects should be considered more.

\section{Future trends}

Nowadays, the micro-nanorobot has achieved some level of success in single cell analysis. It provides much higher manipulation accuracy and stability than manual operation. Thus, the implement of many complex tasks becomes possible, and a lot of exciting new findings have been brought. However, we should realize that the micro-nanorobotic manipulation system still has shortages in many aspects, including low efficient actuation, lacking sensing approaches, high control difficulty, low integration, and it is far from commercialization. A combination of the traditional robotic technique and the micro-nanofabrication technique should be a way to solve the current problems. Next, although there are many micro-nanorobot prototypes, the mathematical model and the control strategy are not mature at the current stage, since it is highly interdisciplinary. Therefore, the cooperation and effort from different research fields is definitely required, including robotic, physics, chemical, biological, and so on. Next, at the small scale, sometimes the manipulation method based on biological and chemical principles is more efficient than the traditional mechanical and electrical method. Therefore, referring biological and chemical principles to improve the current robotic technique should be another trend in the future. Last but not least, extending the application of the micro-nanorobot from laboratory to practical applications should be taken as a significant issue all of the time.

\section{Conclusions}

As the basic unit of life, the single cell receives increasing interest nowadays. The micro-nanorobotic manipulation technique provides an effective approach for single cell analysis. It has been widely used for single cell injection, positioning, characterization, 3D assembly, and other related researches, by which it improves the manipulation accuracy and repeatability and increases the working efficiency greatly. We should realize that despite the fact that the micro-nanorobot has achieved many successes, there are still many challenges that need to be addressed in the single cell analysis field, including the actuating, sensing, controlling, integrating, and commercialization. The future trend of the micro-nanorobot should be much smaller, more efficient, more intelligent, widely applicable, and practical and acceptable by the market.

\section{Competing interests}

The authors declare that they have no competing interests.

\section{Authors' contributions}

YS and TF determined the architectural and contents of the manuscript. YS carried out the data collection and drafted the manuscript. Both authors read and approved the final manuscript. 


\section{Acknowledgement}

This publication is supported by the National Natural Science Foundation of China (61403323), the Early Career Scheme from UGC of Hong Kong (21201314), and the Applied Research Grant of City University of Hong Kong $(6000475,9610300,7200356)$

\section{Author details}

${ }^{1}$ Department of Mechanical and Biomedical Engineering, City University of Hong Kong, Tat Chee Avenue, Kowloon, Hong Kong 99907, China. ${ }^{2}$ School of Mechatronic Engineering, Beijing Institute of Technology, 5 South

Zhongguancun Street, Haidian District, Beijing 100081, China.

\section{Received: 30 July 2014 Accepted: 6 November 2014} Published online: 21 November 2014

\section{References}

1. Alberts B, Johnson A, Lewis J, Raff M, Roberts K, Walter P (2002) Molecular Biology of the Cell. Garland Science, New York

2. Anselmetti D (2009) Single Cell Analysis: Technologies and Applications. Vch Pub, German

3. Brehm-Stecher BF, Johnson EA (2004) Single-cell microbiology: tools, technologies, and applications. Microbiol Mol Biol Rev 68:538

4. Schubert C (2011) The deepest differences. Nature 480:133-137

5. Macaulay IC, Voet T (2014) Single cell genomics: advances and future perspectives. PLoS Genet 10:e1004126

6. Polonsky M, Zaretsky I, Friedman N (2013) Dynamic single-cell measurements of gene expression in primary lymphocytes: challenges, tools and prospects. Brief Funct Genomics 12:99-108

7. Ning L, Liu G, Li G, Hou Y, Tong Y, He J (2014) Current challenges in the bioinformatics of single cell genomics. Frontiers in Oncology 4:7

8. Wang D, Bodovitz S (2010) Single cell analysis: the new frontier in 'omics'. Trends Biotechnol 28:281-290

9. Zheng XT, Li CM (2012) Single cell analysis at the nanoscale. Chem Soc Rev 41:2061-2071

10. Dario P, Guglielmelli E, Allotta B (1994) Robotics in medicine. In: Intelligent Robots and Systems' 94.'Advanced Robotic Systems and the Real World', IROS'94. Proceedings of the IEEE/RSJ/GI International Conference on, Munich, Germany, pp 739-752

11. Dario P, Carrozza MC, Benvenuto A, Menciassi A (2000) Micro-systems in biomedical applications. J Micromech Microeng 10:235

12. Park S, Park J-O (2008) Frontier research program on biomedical microrobot for intravascular therapy. In: Biomedical Robotics and Biomechatronics, 2008. BioRob 2008, 2nd edn. 2nd IEEE RAS \& EMBS International Conference on, Scottsdale, USA, pp 360-365

13. Nelson BJ, Kaliakatsos IK, Abbott JJ (2010) Microrobots for minimally invasive medicine. Annu Rev Biomed Eng 12:55-85

14. Lenaghan SC, Wang Y, Xi N, Fukuda T, Tarn T, Hamel WR, Zhang M (2013) Grand challenges in bioengineered nanorobotics for cancer therapy. IEEE Transactions on Bio-medical Engineering 60:667-673

15. Pepperkok R, Schneider C, Philipson L, Ansorge W (1988) Single cell assay with an automated capillary microinjection system. Exp Cell Res 178:369-376

16. Matsuoka H, Komazaki T, Mukai Y, Shibusawa M, Akane H, Chaki A, Uetake N, Saito M (2005) High throughput easy microinjection with a single-cell manipulation supporting robot. J Biotechnol 116:185-194

17. Zhang Y, Yu LC (2008) Single-cell microinjection technology in cell biology. Bioessays 30:606-610

18. Sun Y, Nelson BJ (2002) Biological cell injection using an autonomous microrobotic system. Int J Robotics Res 21:861-868

19. Lamontagne CA, Cuerrier CM, Grandbois M (2008) AFM as a tool to probe and manipulate cellular processes. Pflügers Archiv European J Physiology 456:61-70

20. Lacal JC, Perona R, Feramisco J (1999) Microinjection: XD-US vol. 1. Springer, Spain

21. Xie Y, Sun D, Liu C, Tse HY, Cheng SH (2010) A force control approach to a robot-assisted cell microinjection system. Int J Robotics Res 29:1222-1232

22. Yanagida K, Katayose H, Yazawa H, Kimura Y, Konnai K, Sato A (1999) The usefulness of a piezo-micromanipulator in intracytoplasmic sperm injection in humans. Hum Reprod 14:448-453

23. Tan Y, Sun D, Huang W, Cheng SH (2008) Mechanical modeling of biological cells in microinjection. NanoBioscience IEEE Transactions on 7:257-266
24. Han S-W, Nakamura C, Kotobuki N, Obataya I, Ohgushi H, Nagamune T, Miyake J (2008) High-efficiency DNA injection into a single human mesenchymal stem cell using a nanoneedle and atomic force microscopy. Nanomedicine 4:215-225

25. Chronis N, Lee LP (2005) Electrothermally activated SU-8 microgripper for single cell manipulation in solution. J Microelectromech Syst 14:857-863

26. Sakar MS, Steager EB, Cowley A, Kumar V, Pappas GJ (2011) Wireless manipulation of single cells using magnetic microtransporters. In: Robotics and Automation (ICRA). IEEE International Conference on, Shanghai, China, pp 2668-2673

27. Arai F, Yoshikawa K, Sakami T, Fukuda T (2004) Synchronized laser micromanipulation of multiple targets along each trajectory by single laser. Appl Phys Lett 85:4301-4303

28. Yue T, Nakajima M, Tajima H, Fukuda T (2013) Fabrication of microstructures embedding controllable particles inside dielectrophoretic microfluidic devices. Int J Adv Robot Syst 10:132

29. Chiou PY, Ohta AT, Wu MC (2005) Massively parallel manipulation of single cells and microparticles using optical images. Nature 436:370-372

30. Beyeler F, Neild A, Oberti S, Bell DJ, Sun Y, Dual J, Nelson B (2007) Monolithically fabricated microgripper with integrated force sensor for manipulating microobjects and biological cells aligned in an ultrasonic field. J Microelectromech Syst 16:7-15

31. Chan H-Y, Li WJ (2003) A thermally actuated polymer micro robotic gripper for manipulation of biological cells. In: Robotics and Automation. Proceedings. ICRA'03. IEEE International Conference on, Taipei, Taiwan, pp 288-293

32. Kim K, Liu X, Zhang Y, Sun Y (2008) Nanonewton force-controlled manipulation of biological cells using a monolithic MEMS microgripper with two-axis force feedback. J Micromech Microeng 18:055013

33. Fatikow S, Rembold U (1996) An automated microrobot-based desktop station for micro assembly and handling of micro-objects. In: EFTA ' 96. Proceedings, 1996 IEEE Conference on. vol.2., pp 586-592

34. Ashkin A, Dziedzic J (1987) Optical trapping and manipulation of viruses and bacteria. Science 235:1517-1520

35. Block SM, Goldstein LSB, Schnapp BJ (1990) Bead Movement by Single Kinesin Molecules Studied With Optical Tweezers. Nature 348:348-352

36. Wang MD, Yin H, Landick R, Gelles J, Block SM (1997) Stretching DNA with optical tweezers. Biophys J 72:1335-1346

37. Block SM, Blair DF, Berg HC (1989) Compliance of Bacterial Flagella Measured With Optical Tweezers. Nature 338:514-518

38. Hu S, Sun D (2011) Automatic transportation of biological cells with a robot-tweezer manipulation system. Int J Robotics Res 30:0278364911413479

39. Castellanos A, Ramos A, Gonzalez A, Green NG, Morgan H (2003) Electrohydrodynamics and dielectrophoresis in microsystems: scaling laws. J Phys D Appl Phys 36:2584

40. Jones TB (2005) Electromechanics of Particles. Cambridge Univ Pr, UK

41. Chang HC, Yeo L (2008) Electrokinetically-Driven Microfluidics and Nanofluidics. Cambridge University Press, USA

42. Hunt T, Westervelt R (2006) Dielectrophoresis tweezers for single cell manipulation. Biomed Microdevices 8:227-230

43. Lee GY, Lim CT (2007) Biomechanics approaches to studying human diseases. Trends Biotechnol 25:111-118

44. Hamill O, Marty A, Neher E, Sakmann B, Sigworth F (1981) Improved patch-clamp techniques for high-resolution current recording from cells and cell-free membrane patches. Pflugers Arch 391:85-100

45. Shen Y, Nakajima M, Kojima S, Homma M, Fukuda T (2011) Study of the time effect on the strength of cell-cell adhesion force by a novel nano-picker. Biochem Biophys Res Commun 409:160-165

46. Ahmad MR, Nakajima M, Kojima S, Homma M, Fukuda T (2011) Buckling nanoneedle for characterizing single cells mechanics inside environmental SEM. Nanotechnology IEEE Transactions on 10:226-236

47. Ahmad MR, Nakajima M, Kojima M, Kojima S, Homma M, Fukuda T (2012) Instantaneous and quantitative single cells viability determination using dual nanoprobe inside ESEM. Nanotechnology IEEE Transactions on 11:298-306

48. Chu YS, Dufour S, Thiery JP, Perez E, Pincet F (2005) Johnson-KendallRoberts theory applied to living cells. Phys Rev Lett 94:28102

49. Yamamoto A, Mishima S, Maruyama N, Sumita M (1998) A new technique for direct measurement of the shear force necessary to detach a cell from a material. Biomaterials 19:871-879

50. Puech P-H, Poole K, Knebel D, Muller DJ (2006) A new technical approach to quantify cell-cell adhesion forces by AFM. Ultramicroscopy 106:637-644 
51. Krieg M, Arboleda-Estudillo Y, Puech P-H, Käfer J, Graner F, Müller DJ, Heisenberg C-P (2008) Tensile forces govern germ-layer organization in zebrafish. Nature Publishing Group 10:429-436

52. Marshall BT, Long M, Piper JW, Yago T, McEver RP, Zhu C (2003) Direct observation of catch bonds involving cell-adhesion molecules. Nature 423:190-193

53. Benoit M, Gabriel D, Gerisch G, Gaub HE (2000) Discrete interactions in cell adhesion measured by single-molecule force spectroscopy. Nat Cell Biol 2:313-317

54. Shen $Y$, Nakajima M, Ridzuan Ahmad M, Kojima S, Homma M, Fukuda T (2011) Effect of ambient humidity on the strength of the adhesion force of single yeast cell inside environmental-SEM. Ultramicroscopy 111:1176-1183

55. Shen Y, Ahmad MR, Nakajima M, Kojima S, Homma M, Fukuda T (2011) Evaluation of the single yeast cell's adhesion to ITO substrates with various surface energies via ESEM nanorobotic manipulation system. NanoBioscience IEEE Transactions on 10:217-224

56. Shen Y, Nakajima M, Kojima S, Homma M, Kojima M, Fukuda T (2011) Single cell adhesion force measurement for cell viability identification using an AFM cantilever-based micro putter. Meas Sci Technol 22:115802

57. Ning W, James PB, Donald El (1993) Mechanotransduction across the cell surface and through the cytoskeleton. Science 260:21

58. Wang K, Sun D (2012) Influence of semiflexible structural features of actin cytoskeleton on cell stiffness based on actin microstructural modeling. J Biomech 45:1900-1908

59. Li M, Liu L, Xi N, Wang Y, Dong Z, Tabata O, Xiao X, Zhang W (2011) Imaging and measuring the rituximab-induced changes of mechanical properties in B-lymphoma cells using atomic force microscopy. Biochem Biophys Res Commun 404:689-694

60. Lam WA, Rosenbluth MJ, Fletcher DA (2007) Chemotherapy exposure increases leukemia cell stiffness. Blood 109:3505-3508

61. Sarah EC, Yu-Sheng J, Qing-Yi L, JianYu R, James KG (2011) Green tea extract selectively targets nanomechanics of live metastatic cancer cells. Nanotechnology 22:215101

62. Wu Y, Sun D, Huang W (2011) Mechanical force characterization in manipulating live cells with optical tweezers. J Biomech 44:741-746

63. Bausch AR, Ziemann F, Boulbitch AA, Jacobson K, Sackmann E (1998) Local measurements of viscoelastic parameters of adherent cell surfaces by magnetic bead microrheometry. Biophys J 75:2038-2049

64. Vogler H, Draeger C, Weber A, Felekis D, Eichenberger C, Routier-Kierzkowska AL, Boisson-Dernier A, Ringli A, Nelson BJ, Smith RS, Grossniklaus U (2013) The pollen tube: a soft shell with a hard core. Plant J 73:617-627

65. Dao M, Lim C, Suresh S (2003) Mechanics of the human red blood cell deformed by optical tweezers. J Mechanics and Physics of Solids 51:2259-2280

66. Haga H, Sasaki S, Kawabata K, Ito E, Ushiki T, Sambongi T (2000) Elasticity mapping of living fibroblasts by AFM and immunofluorescence observation of the cytoskeleton. Ultramicroscopy 82:253-258

67. Cross SE, Jin YS, Rao J, Gimzewski JK (2007) Nanomechanical analysis of cells from cancer patients. Nat Nanotechnol 2:780-783

68. Callies C, Schön P, Liashkovich I, Stock C, Kusche-Vihrog K, Fels J, Sträter JF, Oberleithner H (2009) Simultaneous mechanical stiffness and electrical potential measurements of living vascular endothelial cells using combined atomic force and epifluorescence microscopy. Nanotechnology 20:175104

69. Oberleithner H (2005) Aldosterone makes human endothelium stiff and vulnerable. Kidney Int 67:1680-1682

70. Sarah EC, Yu-Sheng J, Julianne T, Roger W, JianYu R, James KG (2008) AFMbased analysis of human metastatic cancer cells. Nanotechnology 19:384003

71. Shen Y, Nakajima M, Yang Z, Tajima H, Najdovski Z, Homma M, Fukuda T (2013) Single cell stiffness measurement at various humidity conditions by nanomanipulation of a nano-needle. Nanotechnology 24:145703

72. Zhang C, Li P, Liu L, Wang Y, Gao Z, Li G (2014) Development of mechanostimulated patch-clamp system for cellular physiological study. Mechatronics IEEE/ASME Transactions on 19:1138-1147

73. Pampaloni F, Reynaud EG, Stelzer EH (2007) The third dimension bridges the gap between cell culture and live tissue. Nat Rev Mol Cell Biol 8:839-845

74. Cao Y, Vacanti JP, Paige KT, Upton J, Vacanti CA (1997) Transplantation of chondrocytes utilizing a polymer-cell construct to produce tissueengineered cartilage in the shape of a human ear. Plast Reconstr Surg 100:297-302

75. Haycock JW (2011) 3D cell culture: a review of current approaches and techniques. In: 3D Cell Culture. Springer, USA, pp 1-15
76. Klein F, Richter B, Striebel T, Franz CM, Freymann GV, Wegener M, Bastmeyer M (2011) Two-component polymer scaffolds for controlled three-dimensional cell culture. Adv Mater 23:1341-1345

77. Tasoglu S, Diller E, Guven S, Sitti M, Demirci U (2014) Untethered micro-robotic coding of three-dimensional material composition. Nat Commun 5:3124

78. Carletti E, Motta A, Migliaresi C (2011) Scaffolds for tissue engineering and 3D cell culture. In: 3D Cell Culture. Springer, USA, pp 17-39

79. Liu N, Liang W, Liu L, Wang Y, Mai JD, Lee G-B Li WJ (2014) Extracellularcontrolled breast cancer cell formation and growth using non-UV patterned hydrogels via optically-induced electrokinetics. Lab Chip 14:1367-1376

80. Moglia A, Menciassi A, Schurr MO, Dario P (2007) Wireless capsule endoscopy: from diagnostic devices to multipurpose robotic systems. Biomed Microdevices 9:235-243

81. Peyer KE, Zhang L, Nelson BJ (2013) Bio-inspired magnetic swimming microrobots for biomedical applications. Nanoscale 5:1259-1272

82. Kometani R, Morita T, Watanabe K, Hoshino T, Kondo K, Kanda K, Haruyama Y, Kaito T, Fujita J, Ishida M, Ochiai Y, Matsui S (2004) Nanomanipulator and actuator fabrication on glass capillary by focused-ion-beam-chemical vapor deposition. J Vac Sci Technol B 22:257-263

83. Aoyama H, Fuchiwaki O (2001) Flexible micro-processing by multiple microrobots in SEM. In: Robotics and Automation. Proceedings 2001 ICRA. IEEE International Conference on, Seoul, Korea, pp 3429-3434

84. Nogawa K, Kojima M, Nakajima M, Kojima S, Homma M, Fukuda T (2009) Rotational speed control of Na-driven flagellar motor by dual pipettes. NanoBioscience IEEE Transactions on 8:341-348

85. Martel S, Mohammadi M, Felfoul O, Lu Z, Pouponneau P (2009) Flagellated magnetotactic bacteria as controlled MRI-trackable propulsion and steering systems for medical nanorobots operating in the human microvasculature. Int J Robotics research 28:571-582

86. Ding B, Seeman NC (2006) Operation of a DNA robot arm inserted into a 2D DNA crystalline substrate. Science 314:1583-1585

87. Zheng J, Birktoft JJ, Chen Y, Wang T, Sha R, Constantinou PE, Ginell SL, Mao C, Seeman NC (2009) From molecular to macroscopic via the rational design of a self-assembled 3D DNA crystal. Nature 461:74-77

88. Andersen ES, Dong M, Nielsen MM, Jahn K, Subramani R, Mamdouh W, Golas MM, Sander B, Stark H, Oliveira CLP, Pedersen JS, Birkedal V, Besenbacher F, Gothelf KV, Kjems J (2009) Self-assembly of a nanoscale DNA box with a controllable lid. Nature 459:73-76

89. Zhang Z, Olsen EM, Kryger M, Voigt NV, Tørring T, Gültekin E, Nielsen M, Zadegan RM, Andersen ES, Nielsen MM, Kjems J, Birkedal V, Gothelf KV (2011) A DNA tile actuator with eleven discrete states. Angew Chem Int Ed 50:3983-3987

90. Haibo H, Dong S, Mills JK, Li WJ, Shuk Han C (2009) Visual-based impedance control of out-of-plane cell injection systems. Automation Science and Engineering IEEE Transactions on 6:565-571

91. Pillarisetti A, Anjum W, Desai JP, Friedman G, Brooks AD (2005) Force feedback interface for cell injection. In: Eurohaptics Conference, 2005 and Symposium on Haptic Interfaces for Virtual Environment and Teleoperator Systems. World Haptics 2005. First Joint, Pisa, Italy, pp 391-400

92. Mallouk TE, Sen A (2009) Powering nanorobots. Sci Am 300:72-77

93. Xie Y, Sun D, Liu C, Cheng SH, Liu YH (2009) A force control based cell injection approach in a bio-robotics system. In: Robotics and Automation. ICRA'09. IEEE International Conference on, Kobe, Japan, pp 3443-3448

94. Hagiwara M, Kawahara T, Yamanishi Y, Masuda T, Feng L, Arai F (2011) On-chip magnetically actuated robot with ultrasonic vibration for single cell manipulations. Lab Chip 11:2049-2054

\section{doi:10.1186/s40638-014-0021-4}

Cite this article as: Shen and Fukuda: State of the art: micro-nanorobotic manipulation in single cell analysis. Robotics and Biomimetics 2014 1:21. 